\author{
Anna HANUS ${ }^{1}$ \\ Dorota KACZMAREK ${ }^{2}$
}

\title{
ZWISCHEN DEM MEDIALISIERTEN UND MEDIENSKANDAL. DEMONTAGE EINER REPORTER-IKONE
}

\begin{abstract}
Skandale waren und sind weiterhin ein attraktiver thematischer Stoff für Medien, denn einerseits zeigen sie Normbrüche, überführen die vermeintlich Schuldigen der Missetaten und folglich demontieren Anti-Helden. Andererseits geben sie den Journalisten Anlass zur Selbstdarstellung und -werbung, die sich hier in die Rolle der Verbesserer, Normalisierer Moralisierer etc. versetzen. In den gängigen Skandal-Typologien werden etwa medialisierte und Medienskandale unterschieden. Während die ersteren medial lediglich weiterkommuniziert werden, spielen bei den anderen die Medien eine Hauptrolle als Skandalierer. Es scheint, im medialen Kommunikationsbereich hätte man es mit Mischtypen zu tun, die sich irgendwo zwischen den zwei bereits erwähnten Typen platzieren lassen. Am Beispiel des Skandals um die von Artur Domosławski verfasste Kapuściński-Biografie und der Profilierung des Verfassers in der deutschen Presse wird der Versuch unternommen, diesen Mischtyp zu analysieren. Es wird u.a. der Frage nachgegangen, ob die Presse den Skandal für das eigene Publikum vermittelt oder neigt gerade dazu, den ,eigenen' Skandal zu erzeugen. Eines der Hauptziele des Skandals um die erwähnte Biografie war nämlich die mediale Demontage einer gesellschaftlich angesehenen Persönlichkeit wie des Weltreporters Ryszard Kapuściński. Als interessant erweist sich in diesem Kontext zu analysieren, in wieweit die deutsche Presse zu dieser Demontage beiträgt. Ob sie neutral handelt oder eher voreingenommen über die Skandalumstände berichtet, bleibt zu untersuchen.

Schlüsselwörter: Ryszard Kapuściński, Biografie von Domosławski, Medienskandal, HeldenDemontage, Profilierung.
\end{abstract}

\section{EINLEITUNG}

Gesellschaftspolitische Skandale haben paradoxerweise eine spezifische reinigende Macht: Sie bringen Unruhe in öffentlich handelnde Eliten, rufen Entthronisierungsprozesse von ,Helden " hervor oder stürzen gar gesellschaftlich anerkannte Akteure in den Abgrund. Gerade wegen ihres wirklichkeitsverändernden Effekts werden Skandale zu einem begehrenswerten Stoff für Medien, daher tauchen sie in verschiedenen Kommunikationsformen auf, angefangen mit Print- und Online-Presse über Fernsehen bis hin zur Online-Kommunikation: in social media, Foren, Blogs usw. Allerdings darf man behaupten, dass die bevorzugten Kommunikationsformen zur Konstruktion bzw. Medialisierung von Skandalen nach wie vor Pressetexte sind, in denen Skandale von

1 Dr Anna Hanus, Instytut Filologii Germańskiej, Zakład Teorii Komunikacji Językowej, Uniwersytet Rzeszowski, Rzeszów, autor korespondencyjny: e-mail: hanusan@wp.pl

${ }^{2}$ Dr Dorota Kaczmarek, Instytut Filologii Germańskiej, Zakład Językoznawstwa Niemieckiego, Uniwersytet Łódzki, Łódź 
ihrem Anfang an verfolgt und dem Publikum entsprechend auch kommuniziert werden ${ }^{3}$. Aber selbst in jenem Kommunikationsbereich, wo die Meinungskundgebung meist der seriösen Presse zukommt, von der eine gewisse Prominenz erwartet wird, kann man sich des Eindrucks nicht erwehren, dass sich die seriöse von der Boulevardpresse in der Skandalkommunikation, wenn es um die Jagd nach Sensation und Affären geht, nicht allzu sehr unterscheidet. Das, was die Skandal-Berichterstattung in der Klatsch- und Tagespresse immer noch etwas voneinander trennt, ist ein unterschiedlich ausgewogener Einsatz von sprachlichen, nicht-sprachlichen wie auch typographischen Mitteln, von denen die letzteren zwei besonders in den Boulevardblättern mit Vorliebe gebraucht werden. Nicht unberechtigt schreibt diesbezüglich etwa Volmert von der „Inszenierung des »Spektakels« des »Skandals «"“4 deutsche Berichterstattung kennzeichnende) Tendenz fest, nämlich, dass die liberale bzw. linksliberale Presse im Vergleich zur konservativen für mehr Enthüllungen von Skandalen verantwortlich ist. Und das soll etwa damit zusammenhängen, dass die konservativen Redaktionen zur Moralisierung, Belehrung und Theatralisierung neigen, während die liberalen ,volksnah' skandalisieren ${ }^{5}$. Im Allgemeinen lässt sich mit Landwehrbezüglich der Skandalisierung eine weitgehende Boulevardisierung und Tabloidisierung der Medien nicht übersehen ${ }^{6}$, die nicht selten parallel zu ihrer investigativen Arbeit, die bei der Enthüllung eines Skandals gemacht wird, mit kommerziellen Gewinnen, „[...] Verkaufszahlen und Einschaltquoten [...]" rechnen ${ }^{7}$.

Im vorliegenden Beitrag steht der in Polen ausgebrochene Skandal um die umstrittene Biografie von Artur Domosławski über Ryszard Kapuściński u.d.T. Kapuściński nonfiction (2010) und die Übertragung --des Skandals in die deutschen Medien im Fokus. Da es sich also um einen polnische Artefakte betreffenden Skandal handelt, der in der deutschen meinungsbildenden Presse thematisiert wird, scheint von Interesse zu sein, wie sich die Presse dem ausländischen Skandal gegenüber verhält. Kommuniziert sie ihn lediglich dem eigenen Publikum, indem sie sich auf die polnische Berichterstattung beruft oder konstruiert sie eher ihren ,eigenen' Skandal? Wen stellt die deutsche Presse als Skandalierten in den Fokus: den polnischen Weltreporter oder den- Autor der Biografie? Oder beide Akteure? Wie wird der Skandalierte profiliert? Auf diese Fragen versucht der vorliegende Beitrag Antworten zu geben.

\footnotetext{
${ }^{3}$ J. Volmert, ,Flick. Die gekaufte Republik“. Der Skandal, der die Republik erschütterte?, [w:] Skandale. Strukturen und Strategien öffentlicher Aufmerksamkeitserzeugung, red. Bulkow, K., Petersen, C., VS Verlag, Wiesbaden 2003, S. 38.

W. Holly, Die Ordnung des Skandals. Zur diskursanalytischen Beschreibung eines ,Frame am Beispiel der ,CDU-Spendenaffäre', [w:] Politik, Sprache und Glaubwürdigkeit. Linguistik des politischen Skandals, red. Burkhardt, A., Pape, K.,Westdeutscher Verlag, Wiesbaden 2003, S. 57f.

4 J. Volmert, „Flick. Die gekaufte Republik“. Der Skandal, der die Republik erschütterte?, [w:] Skandale. Strukturen und Strategien öffentlicher Aufmerksamkeitserzeugung, red. Bulkow, K., Petersen, C., VS Verlag, Wiesbaden 2003, S. 38 (Hervorhebungen im Orig.).

${ }_{6}^{5}$ Ibidem.

${ }^{6}$ I. Landwehr, Unfundiert, tendenziös und unnötig verletzend. GeenStijl - Das Medium ist der Skandal, [w:] Skandale. Strukturen und Strategien öffentlicher Aufmerksamkeitserzeugung, red. Bulkow, K., Petersen, C., VS Verlag, Wiesbaden 2011, S. 233.

7 J. Volmert, „Flick. Die gekaufte Republik“. Der Skandal, der die Republik erschütterte?, [w:] Skandale. Strukturen und Strategien öffentlicher Aufmerksamkeitserzeugung, red. Bulkow, K., Petersen, C., VS Verlag, Wiesbaden 2003, S. 38.
} 


\section{MECHANISMEN DES PUBLIKMACHENS DER SKANDALE}

Burkhardt differenziert bezüglich der medialen Mechanismen der Enthüllung von Skandalen und der öffentlichen Verhandlungsprozesse über die damit verbundenen Normbrüche zwischen „dem medialisierten Skandal“ und „dem Medienskandal“". Beim medialisierten Skandal spielen die Medien allerdings die Rolle der Vermittler der Skandalinhalte an das Publikum, ohne selbst irgendwelche Details zu einem Skandal aufzubauschen, während im Falle der Medienskandale Redaktion, Journalisten usw. für das Konstruieren und Kommunizieren eines Skandals verantwortlich sind ${ }^{9}$. Hier agieren Medien gegen namentlich bekannte Skandalierte, indem sie den Skandal aufdecken, ihn veröffentlichen und eine gesellschaftliche Empörung darüber produzieren. Die mediale ,Gegenkampagne', wenn sie erfolgreich geführt wird, kann eine neue Ordnung herbeibringen. Für diese ordnende Funktion der Skandale gelten zumindest zwei Szenarien: Ein Szenario nimmt an, dass Skandalierte sich für ihre Missetaten verantworten und die Konsequenzen dafür tragen (müssen). Das zweite Szenario ist mit einer öffentlichen Schuldbefreiung verbunden, wenn der Skandalierte sein Ansehen wiedergewinnen kann. Aus diesem Grund kann man sich der Meinung von Burkhardt nur anschließen:,Medienskandale sind eine eigene Kategorie der Kommunikationspraxis, die ausgelöst durch ein spezifisches journalistisches Narrationsmuster im Zusammenspiel von Informations- und Unterhaltungsmedien die symbolischen Ordnungen von sozialen Systemen qualifizieren und aktualisieren “10.

Dass die mediale Skandalisierung meist politisch handelnde Akteure anvisiert, die darüber hinaus zur Zeit der Skandalisierungsprozesse noch am Leben sind, leuchtet ein. Zum einen sind Ausmaß, Gründe und Folgen eines Skandals für eine konkrete (Teil)Öffentlichkeit von Belang. Zum anderen verbindet diese Öffentlichkeit mit den in den Skandal verwickelten prominenten Akteuren Einfluss- und Statussymbole, die wegen des Skandals beeinträchtigt werden, worauf etwa Weber hinweist ${ }^{11}$. Hinzu kommt, dass bekannte Skandalierte leichter öffentlich der normwidrigen Taten überführt und zu einem Handeln aufgerufen werden. Diesbezüglich schreibt etwa Beckmann von der Polyvalenz der Mehrfachadressierung und der dialogischen Struktur der Skandalkommunikation, in der verschiedene Handlungskomponenten sequenziell verlaufen (z.B. Vorwurf -

${ }^{8}$ S. Burkhardt, Skandal, mediatisierter Skandal, Medienskandal: Eine Typologie öffentlicher Empörung, [w:] Skandale. Strukturen und Strategien öffentlicher Aufmerksamkeitserzeugung, red. Bulkow, K., Petersen, C., VS Verlag, Wiesbaden 2011, S. 133ff. Der Autor nennt als dritten noch den „Skandal ohne Medienberichterstattung“, der nicht zum Gegenstand dieser Analyse gehört (ibidem).

${ }^{9}$ Landwehr schreibt von einer Überstrapazierung des Begriffs Medienskandal, der im Prinzip jeden öffentlichen Verstoß gegen Normen implizieren kann.

I. Landwehr, Unfundiert, tendenziös und unnötig verletzend. GeenStijl - Das Medium ist der Skandal, [w:] Skandale. Strukturen und Strategien öffentlicher Aufmerksamkeitserzeugung, red. Bulkow, K., Petersen, C., VS Verlag, Wiesbaden 2011, S. 227.

${ }^{10}$ S. Burkhardt, Skandal, mediatisierter Skandal, Medienskandal: Eine Typologie öffentlicher Empörung, [w:] Skandale. Strukturen und Strategien öffentlicher Aufmerksamkeitserzeugung, red. Bulkow, K., Petersen, C., VS Verlag, Wiesbaden 2011, S. 134.

${ }^{11}$ P. Weber, Determinanten von Skandalisierung in der politischen Auslandsberichterstattung: Eine empirische Analyse, [w:] Skandale. Strukturen und Strategien öffentlicher Aufmerksamkeitserzeugung, red. Bulkow, K., Petersen, C., VS Verlag, Wiesbaden 2011, S. 113115. 
Rechtfertigung, Vorwurf - Gegenvorwurf usw.) ${ }^{12}$. Auch wenn sich Medien mit den aktuellen, meist den politischen Bereich tangierenden Skandalen bevorzugt beschäftigen, bedeutet das nicht, dass sich mediale Entthronisierungsprozesse nicht auf die einzelnen ,Helden“ als Symbole für Ruhm, Anerkennung usw. beziehen, denn gerade von ihnen wird ein vorbildliches Verhalten erwartet. Aus diesem Grund rücken besonders Fragen nach Moral, Ehre, Wahrhaftigkeit und Glaubwürdigkeit der Skandalierten ins Blickfeld der Medien. Man denke etwa an Karl-Theodor zu Guttenberg und die Plagiatsaffäre um seine Dissertation (2011), in deren Folge er sein Amt als Verteidigungsminister verloren hat. Zu erwähnen sei auch der Skandal um Lech Wałęsa und die Spitzelvorwürfe gegen ihn sowie die seine Vergangenheit als möglicher Geheimagent beschreibenden Bücher etwa von Cenckiewicz. Beide Skandale gelangten an die Presse zwar infolge der Enthüllungen von Dritten, wurden aber medial an die große Glocke gehängt. In solchen Fällen, wo sich die von Burkhardt gezogene Trennung zwischen den medialisierten und Medienskandalen verwischt, kann es sich unserer Ansicht nach um hybride Typen von Skandalen handeln. Für diesen Typ möchten wir die Bezeichnung ,mediale Helden-Demontage 'vorschlagen, die eine Affinität zur Bezeichnung von Otto Graf Lambsdorff „Hinrichtungsjournalismus“ zeigt $^{13}$. Sie impliziert eine Skandalisierung postfactum, d.h. skandalisiert wird eine Person, die sich nicht mehr verteidigen und auf Vorwürfe reagieren kann.

Ein gutes Beispiel für eine mediale Demontage ist etwa der von uns ausgewählte Skandal um den polnischen Weltreporter Ryszard Kapuściński und die nach seinem Tod erschienene Biografie von Artur Domosławski. Im Zentrum des Skandals stehen sowohl das Buch und sein Autor, ferner kontroverse Inhalte der Biografie bezüglich Kapuścińskis als auch eine daraus folgende Diskussion darüber, ob der Reporter trotzalldem eine weltbekannte Autorität, für den Literatur-Nobelpreis nominiert, bleibt oder wegen der im Buch genannten Fakten zu Recht zum Lügner abgestempelt wird. Dieses berücksichtigend kann man sagen, dass der Skandal um Kapuściński für das deutsche Publikum als Fremdskandal nacherzählt wird. Die Medialisierung eines Fremdskandals resultiert u.a. aus der journalistischen Verpflichtung, als Auslandskorrespondenten für das eigene Publikum über relevante fremde Angelegenheiten $\mathrm{zu}$ berichten, worauf etwa Weber in seiner Analyse zu den Einflussfaktoren auf die Berichterstattung über Fremdskandale hinweist ${ }^{14}$. Andererseits werden in der deutschen Presseaus dem Skandal um Kapuściński und der Biografie um Kapuściński Details herausgegriffen, mit denen der ,eigene“ Skandal konstruiert wird. An der Stelle kann man also dem Kommunikationsforscher Kepplinger zustimmen, wenn er in seinem Presseartikel für Die Welt über gegenwärtige Mechanismen der Skandalisierung schreibt: „Skandale sind die demokratische Variante

12 S. Beckmann, Der Skandal - ein komplexes Handlungsspiel im Bereich öffentlicher Moralisierungskommunikation, [w:] Strategien politischer Kommunikation. Pragmatische Analyse, red. Girnth, H., Spieß, C., Erich Schmidt Verlag GmbH \&Co, Berlin 2011, S. 68-71.

13 Vgl. Vom wagnerischen Wahnsinn. Die Republik im Aufruhr, [w:] Der Spiegel Nr. 2, 2007. Abrufbar unter http://www.spiegel.de/spiegel/print/d-50110014.html, S. 57 (10.03.2015).

${ }^{14} \mathrm{P}$. Weber, Determinanten von Skandalisierung in der politischen Auslandsberichterstattung: Eine empirische Analyse, [w:] Skandale. Strukturen und Strategien öffentlicher Aufmerksamkeitserzeugung, red. Bulkow, K., Petersen, C., VS Verlag, Wiesbaden 2011, S. 105127. 
von Schauprozessen“15. Und da zur Inszenierung des Skandals um Kapuściński die deutsche meinungsbildende Presse vor der Jagd nach Sensation nicht zurückscheut, greift sie gerne zu solchen Mechanismen, die die Skandalisierung wirksam werden lassen. Auf diese Aspekte wollen wir in Kap. 4 kurz eingehen.

\section{HINTERGRÜNDE UND BESTIMMUNG DES SKANDALS UM DAS BUCH KAPUŚCIŃSKI NON-FICTION}

Die Biografie Kapuściński non-fiction, die 2010, drei Jahre nach dem Tod von Ryszard Kapuściński, auf den Markt kam, war nicht die erste Arbeit zu dem Thema. Zu diesem Zeitpunkt waren bereits mehrere Kapuściński-Biografien publiziert worden. Keine hat jedoch für so viel Aufsehen gesorgt wie die von Artur Domosławski, einem anerkannten Publizisten der linksliberalen Tageszeitung Gazeta Wyborcza. Domosławski war nämlich, wie er sich selbst bezeichnete, ein Freund und Schüler von Kapuściński und brachte eine Arbeit heraus, die die ganze Wahrheit über das Leben und Werkeiner der größten Kapazitäten Polens des 20. und 21. Jahrhunderts im Bereich politischer Reportage vermitteln sollte. Das Erscheinen des Buches löste eine erregte Debatte in den polnischen Medien aus. Zu Wort meldeten sich Journalisten, Reporter, Politiker, Schriftsteller, ja sogar prominente Vertreter der katholischen Kirche. Die Biografie verkaufte sich wie warme Semmeln und die Startauflage von 45000 Exemplaren war innerhalb von wenigen Tagen restlos verkauft, sodass die gleiche Zahl noch einmal nachgedruckt werden musste.

Was also war das Geheimnis des Erfolgs von Domosławski? Was hat die Öffentlichkeit so erschüttert, dass eine fast gesamtpolnische Debatte über sein Werk ausgebrochen ist?

Der Biograf hat eine durchaus kontroverse Arbeit herausgebracht, in der er an dem bisherigen Bild des berühmten Reporters kräftig kratzt, indem er u.a. die Glaubwürdigkeit des ,Meisters der Reportage“ in Frage stellt.

Das kontroverse Thema erschien auch der ausländischen Presse interessant und wurde gleich in mehreren Presseredaktionen der Welt wahrgenommen. Warum ist aber das Thema auf ein so großes Interesse im Ausland gestoßen, warum der ohnehin fremde Skandal so gern aufgegriffen worden?

Ryszard Kapuściński war kein durchschnittlicher Reporter, seine Bücher wurden in beinahe der ganzen Welt gelesen. Übersetzt wurden sie in über 36 Sprachen. Kapuściński hat Jahrzehnte lang aus den Gebieten berichtet, die sich im Umbruch befanden, von Umstürzen, Befreiungskämpfen, Aufbrüchen. Oft war er, wie es mehrere Reporter mit Neid gestanden, „der einzige schreibende Zeuge“"16. Kapuściński hat vor allem aber das Genre Reportage revolutioniert, weswegen auch von der polnischen Schule der Reportage gesprochen wird und er nobelpreisverdächtig war.

15 H. M Kepplinger, Die psychologischen Gesetze des modernen Skandals, [w:] http://www.welt.de/debatte/kommentare/article13901048/Die-psychologischen-Gesetze-desmodernen-Skandals.html, 2012 (04.12.2016).

${ }^{16}$ So hat sich über Kapuściński beispielsweise Bartholomäus Grill geäußert (Die Zeit, Nr. 10, 04.03.2010). 


\section{SKANDAL UM KAPUŚCIŃSKI NON-FICTION \\ IN DER DEUTSCHSPRACHIGEN PRESSE}

Wohl wegen der großen internationalen Resonanz seiner Texte ist der Skandal um Kapuściński in der deutschsprachigen meinungsbetonten Presse zu einem prominenten Thema geworden.

In dem Zusammenhang erscheint es von Belang, sich mit der Frage auseinanderzusetzen, wie deutsche Printmedien auf die fremde Aufregung um das Buch reagiert haben, ob sie über den Eklat lediglich berichtet und auf polnische Quellen hingewiesen, oder vielleicht ihren ,eigenen Skandal' konstruiert haben. Werden wir dann somit mit dem medialisierten Skandal oder dem Medienskandal im Sinne von Burkhardt konfrontiert ${ }^{17}$ ?

Bevor wir uns aber der Hauptfrage zuwenden, wäre es sinnvoll sich für einen Augenblick bei dem Skandalierten aufzuhalten. Aus mehreren Texteinleitungen, zuweilen auch Artikelüberschriften geht nämlich hervor, dass die kontroverse Biografie zum Skandalauslöser wurde. Der Autor der Veröffentlichung wäre somit der Skandalierte:

(1) Gabriele Lesser, Kapuścińskis-Skandal-Biografie ${ }^{18}$. Dichtung und Wahrheit in Polen (Die Tageszeitung 2010)

(2) Doch darf ein Journalist lügen, um so einer übergeordneten Wahrheit zum Sieg zu verhelfen? Darf er Personen und Orte erfinden, um seine Reportage in eine Allegorie der Macht zu verwandeln? In Polen haben diese Fragen einen Skandal ausgelöst, galt Kapuściński doch als "Jahrhundertreporter". (Gabriele Lesser, Kapuścińskis-Skandal-Biografie, Die Tageszeitung 2010)

(3) Dem 2007 verstorbenen polnischen Schriftsteller Ryszard Kapuścinski haben seine Anhänger bereits zu Lebzeiten den Ehrentitel «Kaiser der Reportage» verliehen. Kapuścinski kennt und schätzt man in aller Welt. Offenbar wusste man aber $\mathrm{zu}$ wenig von ihm. Eine Kapuścinski-Biografie des polnischen Journalisten - und nach eigenen Aussagen glühenden Kapuścinski-Verehrers Artur Domosławski erschien so skandalträchtig, dass der Krakauer ZnakVerlag sich von der Veröffentlichung zurückzog, während die Witwe des Porträtierten gerichtliche Schritte einleitete (Martin Sander, Kapuściński entzaubert)

Wenn man dann die in den deutschsprachigen Printmedien veröffentlichten Texte genauer analysiert, muss man mit Erstaunen feststellen, dass der Skandalierte gar nicht der Verfasser der Kapuściński-Biografie ist, und auch nicht über sein Buch, seine Leistung, seine Art und Weise der biografischen Schilderung diskutiert wird. In den Fokus der kritischen Auseinandersetzung rückt der ,Meister der Reportage' selbst. Sein Lebenslauf wird kommentiert, seine Entscheidungen, seine Reportagen, seine Reporterethik werden kritisch beurteilt, ja sogar angeprangert.

In die Irre wird der Forscher ebenfalls geführt, wenn er davon ausgeht, dass die Artikel ein gutes Beispiel für den medialisierten Skandal sind. Und das könnte er anhand von mehreren Belegen der Schilderung des polnischen Skandals aus der Fremdperspektive schlussfolgern:

17 S. Burkhardt, Skandal, mediatisierter Skandal, Medienskandal: Eine Typologie öffentlicher Empörung, [w:] Skandale. Strukturen und Strategien öffentlicher Aufmerksamkeitserzeugung, red. Bulkow, K., Petersen, C., VS Verlag, Wiesbaden 2011.

${ }^{18}$ Alle Hervorhebungen in Belegen, wenn nicht anders vermerkt, stammen von den Verfasserinnen. 
(4) War Ryszard Kapuściński ein Lügner, ein schreibender Hochstapler? Hat es dieser Mann, der in Polen den Status einer Ikone genießt und vor seinem Tod zum »Reporter des Jahrhunderts« gekürt wurde, mit den Tatsachen nicht so genau genommen? Das zumindest behauptet Artur Domosławski, ein junger Journalist der linksliberalen Tageszeitung "Gazeta Wyborcza« und einst Ziehsohn Kapuścińskis. (Knut Krohn, Der »Reporter des Jahrhunderts" Hochstapler, Lügner oder doch ein Künstler?, Tagesanzeiger 2010)

(5) Nun ist eine Biografie erschienen, die das Denkmal demontiert. Artur Domosławski, ein Vertrauter Kapuścińskis, behauptet, der Weltreporter habe in seinen Reportagen nicht nur kräftig manipuliert, sondern Geschichten erdichtet und sogar Begegnungen erfunden, zum Beispiel mit Che Guevara oder Patrice Lumumba. Kapuściński - ein Lügenbaron? So weit geht der Biograf nicht, aber er weist nach, dass es sein Held mit den Grenzen zwischen Literatur und Journalismus nicht so genau nahm. Er war ein Borderline-Schreiber, der bisweilen Fakten und Fiktionen vermischte. (Bartholomäus Grill, Ryszard Kapuściński. An der Borderline, Zeit online 2010)

(6) Die erste Biografie des vor drei Jahren gestorbenen polnischen Starreporters Ryszard Kapuściński sorgt in Polen für Aufregung. Verleger aus Frankreich, Italien und Spanien hätten auf eine Veröffentlichung verzichtet, nachdem die Witwe des Journalisten gedroht habe, ihnen Rechte auf Neuauflagen der Bücher von Kapuściński zu verweigern, berichtete die Zeitung „Rzeczpospolita“ am Samstag. Alicja Kapuscinska hatte vorher auf dem Rechtsweg versucht, das Erscheinen der Biografie in Polen zu stoppen. Ein Gericht wies aber vor einer Woche ihre Beschwerde zurück. (Umstrittene Biografie, Die Berliner Literaturkritik 2010)

Eine eingehende Analyse des gesammelten Korpus bestätigt allerdings das Gegenteil. Wenn nämlich die Textinhalte der in Polen und der in den deutschsprachigen Printmedien veröffentlichen Artikel sowie die der Biografie gegenüberstellt werden, lassen sich viele Divergenzen und Widersprüchlichkeiten aufdecken. Die formal-stilistische Form des Berichtens, wenn es um Pressetexte geht, wird zwar beibehalten, jedoch stehen Inhalte nicht selten zu den in den polnischen Medien publizierten Aussagen, den vorgebrachten Tatsachen, ja sogar zu dem Buch von Domosławski selbst im Widerspruch. Man hat den Eindruck, die einzelnen deutschen Journalisten seien um einiges weiter über die in Polen erschienenen Texte, samt dem Buch von Domosławski, hinausgegangen, indem sie manche Fakten einfach ohne Überprüfung überinterpretieren bzw. gar frei interpretieren. Im Extremfallkann man sogar annehmen, sie hätten die Informationen extra verklärt, um ihre Leserschaft zu frappieren (vgl. Belege 7-9).

(7) Jetzt also auch Ryszard Kapuściński. Sein Biograph Artur Domosławski hat den wohl berühmtesten polnischen Reporter des 20. Jahrhunderts soeben der Fälschung überführt. Anders als von Kapuściński selbst behauptet, habe dieser weder Che Guevara gekannt, noch sei er je dem kongolesischen Freiheitskämpfer und späteren Premierminister Patrice Lumumba begegnet. (Stephan Ruß-Mohl, Reporter-Legenden: Ryszard Kapuścińskis»Lebenslüge«, Carta 2010)

(8) Zum Beispiel weist Domosławski nach, dass Kapuściński Che Guevara nicht gekannt und den kongolesischen Rebellen Patrice Lumumba nicht getroffen haben kann. Klappentexte seiner Bücher behaupten das aber, und seine 
Artikel klingen auch so. "Übertrieben" sei auch eine Episode, nach der Kapuściński im Kongo zum Tode durch Erschießen verurteilt worden sein soll. (Jan Puhl, Kapuściński-Biografie Weltreporter mit Wahrheitsproblem, Der Spiegel 2010).

(9) So habe Kapuściński Revolutionsführer Che Guevara gar nicht gekannt, weist Domosławski in akribischer Recherche nach. Auch seine Treffen mit dem kongolesischen Rebellen Patrice Lumumba seien erfunden, ebenso wie die legendäre Episode seiner Beinaheerschießung durch ein Exekutionskommando - dabei hatte der Reporter doch immer wieder behauptet, er könne nur über das schreiben, was er auch wirklich selbst gesehen habe. Kapuściński habe bewusst an seiner eigenen Legende gebastelt und sich im Lichte der berühmten Namen gesonnt, sagt Domosławski dazu.

Doch habe es noch mehr Ungereimtheiten im Leben des Journalisten gegeben. So habe er sich gegen Ende des Regimes in Polen gerne öffentlich als Gegner des Kommunismus inszeniert, doch sei er in jüngeren Jahren ein überzeugter Fahnengänger gewesen. Mitte der 1970er-Jahre habe Kapuściński sogar in Angola mit der Waffe in der Hand kommunistische Gruppen unterstützt und sich in der Heimat als Informant des berüchtigten polnischen Geheimdienstes anwerben lassen. Allerdings, wirft Domosławski ein, habe er dabei keinem geschadet. (Knut Krohn, War die polnische Ikone ein Reporter oder ein Lügner?, Tagesanzeiger 2010)

Dafür, dass die in den deutschsprachigen Artikeln veröffentlichten, Aufsehen erregenden Mitteilungen mit den in Polen diskutierten Tatsachen und Aussagen im Widerspruch stehen, gibt es mehrere Beweise. Selbst in der kontroversen Biografie wird nicht behauptet, Kapuściński habe von der Bekanntschaft mit Che Guevara oder Lumumba erzählt, geschweige denn sich damit gebrüstet (vgl. Beleg 10). Auch bezüglich der genannten Klappentexten handelt es sich nicht um mehrere Beispiele, sondern um eine Einzelnotizim Klappentext der englischen Ausgabe von Wojna futbolowa (The Soccer War, Eichborn 2000) [dt. Der Fußballkrieg], die Kapuściński nicht rechtzeitig korrigieren ließ (vgl. Beleg 10). Der Autor der Notiz war darüber hinaus nicht der Reporter, sondern der Verleger selbst. Dafür, dass Kapuściński ,selbst behauptet‘ (Beleg 7) bzw. ,in seinen Artikeln' angedeutet habe (Beleg 8), er habe Che Guevara und Lumumba getroffen, führen die Autoren der Artikel keine Belege an. (Unseres Wissens gibt es keine Artikel und keine aufgenommenen Interviews mit Kapuściński, die das beweisen könnten). Auch die sensationelle Nachricht, der Starreporter habe sich in Polen ,gerne öffentlich als Gegner des Kommunismus inszeniert“ (Beleg 9), ist als frei erfunden zu klassifizieren. Nicht mal Domosławski hat das behauptet. In der Biografie finden sich mehrere Stellen, die gerade das Gegenteil beweisen.

(10) Legenda powstała za sprawą noty na okładce angielskiego wydania Wojny futbolowej: „Przyjaźnił się z Che Guevarą w Boliwii, z Salvadorem Allende w Chile i Patrice'em Lumumbą w Kongu” (...) W szkicu Guevara i Allende z tomu Chrystus z karabinem na ramieniu Kapuściński nie pisze ani słowem o tym, że któregoś z nich spotkał osobiście. Nie ma cienia sugestii, że kiedykolwiek rozmawiali, o czym dziennikarze zazwyczaj spieszą donieść w pierwszych słowach. (Artur Domosławski, Kapuściński non-fiction, Świat Książki, Warszawa, 2010) 
Darüber hinaus sind in dem Korpus mehrere Belege $\mathrm{zu}$ finden, in denen aus der Eigenperspektive der Artikelautoren berichtet und kommentiert wird. Es werden eigene Beobachtungen übermittelt und eigene Urteile abgegeben (vgl. Belege 11, 12).

(11) In seinen weltbekannten Reportagen legte Ryszard Kapuściński den Helden erfundene Aussagen in den Mund. Auch die Legende, er habe Che und den afrikanischen Freiheitshelden Lumumba getroffen, stimmt nicht. (Claudio Habicht, Der Starreporter, der es mit der Wahrheit nicht so genau nahm, Tagesanzeiger 2010).

(12) Schon vor seinem Erscheinen hat das Buch in Polen eine erbitterte Debatte ausgelöst. Dabei bezweifelt niemand, dass die von Domosławski zu Tage geförderten Fakten nicht stimmen könnten. (Gabriele Lesser, KapuścińskisSkandal-Biografie Dichtung und Wahrheit in Polen, Die Tageszeitung 2010)

Leider sind in den Artikeln keine Verweise darauf zu finden, woraus die Textautoren schließen, Kapuściński habe gelogen und seinen „Helden erfundene Aussagen in den Mund“ gelegt. Überraschend klingt auch die Äußerung von Gabriele Lesser über die Überzeugung der gesamten polnischen Leserschaft, „dass die von Domosławski zu Tage geförderten Fakten“" unstrittig wären (Beleg 12). In den polnischen Printmedien sind zu der Zeit mehrere Presseartikel erschienen, die die Behauptung von Lesser widerlegen ${ }^{19}$.

Aus den oben präsentierten Belegen und Kommentaren mit entsprechenden Beweisen geht hervor, dass in der deutschsprachigen Presse über den Fremdskandal häufig nicht unvoreingenommen und eher unsachlich berichtet wird. Oft wird aus der Perspektive des Biografen geurteilt - von der Annahme ausgehend, dass das, was Domosławski in seinem Buch behauptet (und Vieles wird in dem Buch lediglich angedeutet), auch wahr ist (vgl. etwa hat den wohl berühmtesten polnischen Reporter des 20. Jahrhunderts soeben der Fälschung überführt, weist Domostawski in akribischer Recherche nach, Zum Beispiel weist Domosławski nach, dass...). Die Hauptvorwürfe des Biografen werden übernommen, anstatt dass die einzelnen Artikelautoren selbst den Behauptungen auf den Grund gehen. Mehrmals werden die Aussagen von Domosławski auch frei interpretiert, ja sogar verdreht, was an ausgewählten Beispielen gezeigt wurde.

Nicht selten wird auch in der deutschsprachigen Presse aus der Selbstperspektive eines Experten berichtet, ohne jedoch auf Beweismittel zu verweisen, was auf frei Erfundenes vermuten lässt.

Obgleich in mehreren Artikeln die Form des Berichtens beibehalten ist, werden in vielen die Stimmen der polnischen Journalisten und Intelektuellen ${ }^{20}$ contra Domosławski ,übersehen', was im Falle der Berichte als zuverlässige Informationsquelle zu erwarten wäre. Stattdessen werden in fast allen ,Berichterstattungen' die den Skandal abschwächenden Aussagen von Experten ausgelassen. Aus all dem lässt sich

\footnotetext{
${ }^{19}$ Vgl. etwa E. van den Bergen-Makała, Mission impossible Domostawskiego, Gazeta Wyborcza 2010, S. Popowski, Ta ksiązka boli. A jeszcze bardziej dyskusja, która rozpętała... Gazeta Wyborcza 2010, I. Wojciechowska, Punctum, Gazeta Wyborcza 2010.

${ }^{20}$ Gazeta Wyborcza hat eine gesamtpolnische Debatte zur Veröffentlichung der Biografie von Ryszard Kapuściński initiiert, an der alle Intellektuellen aus In- und Ausland teilnehmen konnten. $\mathrm{Zu}$ Wort meldeten sich Politiker, Wissenschaftler, Übersetzer, darunter auch Kapuścińskis Übersetzer, Literaten, Journalisten, Auslandskorrespondenten etc. Erstaunlicherweise wurden in Berichten und Kommentaren der deutschsprachigen Journalisten lediglich die KapuścińskiContrastimmen berücksichtigt.
} 
schlussfolgern, dass der polnische Skandal nicht sachlich übermittelt, sondern für das deutsche Publikum neu inszeniert wurde.

\section{DAS SPRACHLICHE PROFILIEREN DES SKANDALIERTEN-BILDES}

Medien kommt, und zwar zurecht, eine prominente Rolle zu, das sprachliche Bild der wahrnehmbaren Wirklichkeit massenmedial zu präsentieren und zu distribuieren. Oft wird aber die Wirklichkeit, da sie immer eine interpretierbare Wirklichkeit darstellt, nicht nur massenmedial kolportiert, sondern auch konzeptualisiert, ja sogar konstruiert. Medien verfügen über die Macht, einen über die Nacht zu einem Medienstar zu machen, aber auch die Reputation einstmals angesehener Menschen $\mathrm{zu}$ vernichten, gesellschaftlich anerkannte Akteure in den Abgrund zu stürzen. Daher ist es von Relevanz zu fragen, wie Medien die wahrnehmbare Wirklichkeit erzeugen, indem sie Profilbilder von öffentlich bekannten Persönlichkeiten medial gestalten und verbreiten und in unserem Fall, wie der Skandalierte, Ryszard Kapuściński, in den deutschsprachigen Printmedien profiliert wird sowie welche Konsequenzen, im Sinne der Beeinflussung von Denkmustern und der Weltwahrnehmung, das für ihn wohl haben könnte; dass die Folgen für sein Leben allerdings nicht überprüft werden können, leuchtet ein.

Bei der Profilierung des sprachlichen Bildes von Kapuściński wird auf eine der Methoden der linguistischen Diskursanalysezurückgegriffen. Die Analyse konzentriert sich auf die Untersuchung von Nominationen (berücksichtigt werden dabei sowohl einzelne Lexeme, als auch Mehrworteinheiten) und Prädikationen, anhand welcher, als spezifischen sprachlichen Darstellungsmitteln, das Bild des ,Jahrhundertreporters' erstellt wird. Mit Nominationen und Prädikationen wird der Skandalierte benannt und bewertet sowie mit Prädikationen in seinen Rollen bewertet. Wie wird dann somit der Skandalierte sprachlich konzeptualisiert?

Erstaunlich viele Nominationen, die sich auf Kapuściński beziehen, weisen ein eindeutig positiv wertendes Profil auf, indem sie auf seine journalistische Leistung und seinen internationalen Ruhm hinweisen: konkurrenzloser Reporterkönig, Kaiser der Reportage, Jahrhundertreporter, Ikone, Reporter des Jahrhunderts, Starreporter, Journalist des Jahrhunderts, Nationalheld, Idol, Guru, Meister, der meistgelesene polnische Buchautor, Reporter-Legende, Meisterjournalist, Vorbild von Generationen polnischer Journalistenkollegen, Großmeister des polnischen Journalismus, der hoch gekürte Journalist, Gründevater der ,polnischen Schule der Reportage“, Vorbild, polnische Ikone, Kosmopolit. Diese werden jedoch nicht zum Rühmen der fachlichkünstlerischen Leistung der Reporterlegende verwendet. In dem Zusammenhang kommt ihnen eine durchaus differente Funktion zu. Sie werden dazu benutzt, das Bild von Kapuściński zu polarisieren und sein Ansehen, seine Anerkennung mit den von Domosławski zu Tage geförderten, sensationellen ,bloßstellenden Missetaten " der Reporterikone zu kontrastieren (vgl. Belege 13, 14). Dieses Loben soll also im Endeffekt dazu benutzt werden, das Denkmal zu demontieren, das bisher ,gefälschte KapuścińskiBild‘ zu revidieren, zu verifizieren und zu dementieren. Als Beispiele mögen folgende Kontrastierungen gelten, in denen negativ wertende Nominationen zum Tragen kommen: Hochstapler, Lügner, Verbrecher, Angeklagter, schreibender Hochstapler, Fälscher. Hin und wieder wird auf Kapuścińskis ,Verbrechen' direkt hingewiesen. So wird einerseits Ikone mit Lügner gleichgesetzt; an anderen Textstellen tauchen neben Kaiser der Reportage etwa Hochstapler, schreibender Hochstapler usw. auf. 
(13) Dem 2007 verstorbenen polnischen Schriftsteller Ryszard Kapuścinski haben seine Anhänger bereits zu Lebzeiten den Ehrentitel «Kaiser der Reportage» verliehen. Kapuścinski kennt und schätzt man in aller Welt. Offenbar wusste man aber $\mathrm{zu}$ wenig von ihm. (...) Gleichwohl zeichnet die gründlich recherchierte Biografie von Domosławski genauer nach, wie Kapuścinski als Reporter der polnischen Nachrichtenagentur PAP mit dem kommunistischen Sicherheitsdienst zusammenarbeitete. Man erfährt, warum der Autor des «Fussballkriegs» gelegentlich von der Schusswaffe Gebrauch machte, statt zu beobachten, und weshalb er politisch unbequeme Wahrheiten unterdrückte. Zum differenzierten Bild gehört auch: Kapuścinski hat Revolutionäre wie Che Guevara oder Patrice Lumumba nie getroffen - anders als es Klappentexte seiner Bücher suggerieren. (Gerhard Gnauck, Wie kann man Che Guevara treffen, wenn der schon tot war?, Die Welt 2010).

(14)Die Polen haben Kapuściński nicht vergessen, dass er unter dem kommunistischen Regime ein Fenster zur Welt aufgestoßen hatte und seinen Landsleuten erzählte, was jenseits des Eisernen Vorhangs zu sehen war. Kapuściński ist neben Stanislaw Lem der meistgelesene polnische Buchautor im Ausland. Und nun sollen zentrale Bestandteile seines abenteuerlichen Lebenslaufes erlogen sein? Das weist Domosławski in der Tat in akribischer Recherche nach. So habe Kapuściński Revolutionsführer Che Guevara nicht persönlich gekannt. Und auch seine Treffen mit dem kongolesischen Rebellen Patrice Lumumba seien frei erfunden. Ebenso die legendäre BeinaheErschießung durch ein Exekutionskommando - dabei hatte der Reporter doch immer wieder behauptet, er könne nur über das schreiben, was er auch wirklich selbst gesehen habe. (Knut Krohn, Der «Reporter des Jahrhunderts» Hochstapler, Lügner oder doch ein Künstler?, Tagesanzeiger 2010)

Eine weitere Gruppe von positiv wertenden Nominationen stellen diese dar, die dazu gebraucht werden, ironisch über die journalistische Leistung von Kapuściński auszusagen. Es wird darauf hingedeutet, Kapuścińskis Texte seien keine richtigen Reportagen, sie gehörten eher in das Regal mit Reiseromanen: reisender Literat, Meistererzähler, einer der größten polnischen Reiseschriftsteller des 20. Jahrhunderts, literarischer „Fensteröffner“, literarische Ikone, Meister der literarischen Reportage. Den ironischen Gebrauch der positiv wertenden Nominationen veranschaulicht ein Auszug aus dem Artikel von Bartholomäus Grill (vgl. Beleg 15):

(15) Der Fall Ryszard Kapuściński lehrt: Es gibt keinen objektiven Journalismus. Dennoch gilt jenseits des hermeneutischen Zweifels das Gebot der journalistischen Wahrhaftigkeit: Wir wissen nicht, ob ein Ereignis tatsächlich so war, aber wir beschreiben nach bestem Wissen und Gewissen, wie es gewesen sein könnte. Ryszard Kapuściński verletzte dieses Gebot, er ist entzaubert als unbestechlicher Chronist. Fortan müssen wir ihn als reisenden Literaten lesen, dessen Erzählungen zwischen Dichtung und Wahrheit oszillieren.(Bartholomäus Grill, Ryszard Kapuściński. An der Borderline, Zeit online 2010)

Anders werden die Schwerpunkte bei Prädikationen verteilt. Ganz selten wird positiv über Kapuściński prädiziert. Die meisten positiven Wertungen nehmen Bezug auf das internationale Ansehen des Reporters und seine außerordentliche Gabe, die Aufmerksamkeit der Leser zu fesseln. Negativ wertende Prädikationen sind dagegen in 
dem deutschsprachigen Korpus reichlich vertreten, so dass sie sich sogar in Themengruppen aufnehmen und Kapuściński in mehreren differenten Rollen auftreten lassen. Stellvertretend seien folgende Belege angeführt:

1. Kapuściński als Lügner und Mundheld

- hat den kongolesischen Rebellen Patrice Lumumba nie getroffen,

- hat Tatsachen erfunden,

- hat sich gegen Ende des Regimes in Polen öffentlich als Gegner des

Kommunismus inszeniert,

- frisierte seine Lebensgeschichte,

2. Kapuściński - kommunistischer Fahnengänger

- lobte Sozialismus,

- war ein überzeugter Kommunist,

- war in jüngeren Jahren ein überzeugter Fahnengänger,

- stand auf Seiten der marxistischen Befreiungsbewegungen,

3. Vertrauter des Sicherheitsdienstes

- nicht gerade zimperlich bei der Wahl seiner politischen Mentoren in der Partei,

- ging Kompromisse mit der Macht ein,

4. Kapuściński

- kein echter Reporter, der die Wirklichkeit mit der Phantasie vermischte,

-hat immer wieder die „Grenzen zwischen Reportage und Literatur"

überschritten.

\section{SCHLUSSBEMERKUNG UND AUSBLICK}

Aus dieser exemplarischen, linguistisch orientierten Analyse der pressemedial verwendeten Mechanismen der Fremdskandalisierung lässt sich unseres Erachtens sowohl das für die Medienskandale Typische und Untypische, was die Fokussierung auf eine hybride Mischvariante der Skandale rechtfertigen sollte, ableiten. Sicherlich sind an den Thematisierungen des Skandals um Ryszard Kapuściński und der Biografie von Artur Domosławski in der deutschsprachigen Presse keine für die Skandalkommunikation sonst charakteristischen Verlaufsphasen (Latenz-, Aufschwung-, Abschwung- und Rehabilitationsphase) erkennbar, von denen etwa der hier mehrmals erwähnte Burkhardt schreibt ${ }^{21}$. Meist beziehen sich die Textautoren in ihren Berichten zeitlich auf den Höhepunkt des Skandals, nämlich die Veröffentlichung des kontroversen Buches und Domosławskis Entthronisierungsversuche, Kapuścińskis Ruf nachträglich zu demontieren. Wegen der Unmöglichkeit, den skandalierten Weltreporter persönlich und direkt zu beschuldigen, verzichten die Journalisten wohl auf die typischen kommunikativen Skandal-Handlungen, wie etwa Entrüstungs- und Moralisierungshandlungen; stattdessen betreiben sie eine gewisse ,Gegenkampagne“ gegen den Reporter, bei der sie allerdings ziemlich einseitig vorgehen. Das erinnert mehr an einen „Schauprozess“ als an eine zuverlässige Berichterstattung, von der etwa der deutsche, an diesem Skandal interessierte

${ }^{21}$ S. Burkhardt, Medienskandale. Zur moralischen Sprengkraft öffentlicher Diskurse. Köln 2006, S. 204.

S. Burkhardt, Skandal, mediatisierter Skandal, Medienskandal: Eine Typologie öffentlicher Empörung. [w:] Skandale. Strukturen und Strategien öffentlicher Aufmerksamkeitserzeugung, red. Bulkow, K., Petersen, C., VS Verlag, Wiesbaden 2011, S. 142. 
Leser erwarten könnte, Sichtweisen aller Seiten kennen zu lernen. Das scheinen die deutschen Kommentatoren nicht sicherzustellen.

Als untypisch für Medienskandale gelten ferner fehlende Emotionsbekundungen seitens der Journalisten, die bei der Skandalisierung gerne zu verschiedenen sprachlichen und nicht-sprachlichen Mitteln greifen, mit denen sie Emotionen zum Ausdruck bringen. An den Belegen fällt auf, dass sich die Textautoren diesbezüglich eher an Sachverhalten und -ihrer Meinung nach - an Fakten orientieren, und dass sie weniger dazu neigen, emotionsgeladene Werturteile zu formulieren.

Nicht zu übersehen sind allerdings die von uns aufgezeigten, sich in den Pressetexten wiederholenden Profilierungen des Reporters, also Prädikationen und Nominationen, die größtenteils negative Wertungen sind. Kommen positive Nominationen von Kapuściński zum Ausdruck, wird ihr medialer Wert durch Kontraste deutlich abgeschwächt, nach der Regel: Wer war Kapuściński einst? Wer/was ist er nach der Veröffentlichung des Buches geworden? In diesem Sinne gehen die Textautoren dem Prinzip der Negativität nach, die nach Weber ,als wichtigstes inhaltliches Merkmal“ von Skandalinszenierungen gilt ${ }^{22}$.

Ein typisches Merkmal der Skandalisierung ist ihre persuasive Wirkung. Die deutschen Kommentatoren lassen jedoch den potenziellen Rezipienten ihrer Texte keinen freien Raum für Interpretationen, wenn sie ihnen suggestive Bilder von Kapuściński liefern.

Da, wie aus der obigen Zusammenfassung ersichtlich, keine eindeutige Zuordnung des Skandals um Ryszard Kapuściński und seiner Thematisierung in der deutschen Presse entweder zu medialisierten oder Medienskandalen möglich zu sein scheint, rechtfertigt das die Annahme, dass man es hier mit einem Mischtyp, eben einer Helden-Demontage, zu tun hat.

\section{LITERATUR}

[1] Beckmann, S., Der Skandal - ein komplexes Handlungsspiel im Bereich öffentlicher Moralisierungskommunikation, [w:] Strategien politischer Kommunikation. Pragmatische Analyse, red. Girnth, H., Spieß, C., Erich Schmidt Verlag GmbH \&Co, Berlin 2011, S. 61-78.

[2] Burkhardt, S., Medienskandale. Zur moralischen Sprengkraft öffentlicher Diskurse, Köln 2006.

[3] Burkhardt, S., Skandal, mediatisierter Skandal, Medienskandal: Eine Typologie öffentlicher Empörung, [w:] Skandale. Strukturen und Strategien öffentlicher Aufmerksamkeitserzeugung, red. Bulkow, K., Petersen, C., VS Verlag, Wiesbaden 2011, S. 131-156.

[4] Holly, W., Die Ordnung des Skandals. Zur diskursanalytischen Beschreibung eines ,Frame‘ am Beispiel der, CDU-Spendenaffäre‘, [w:] Politik, Sprache und Glaubwürdigkeit. Linguistik des politischen Skandals, red. Burkhardt, A., Pape, K.,Westdeutscher Verlag, Wiesbaden 2003, S. 47-68.

\footnotetext{
${ }^{22} \mathrm{P}$. Weber, Determinanten von Skandalisierung in der politischen Auslandsberichterstattung: Eine empirische Analyse. [w:] Skandale. Strukturen und Strategien öffentlicher Aufmerksamkeitserzeugung, red. Bulkow, K., Petersen, C., VS Verlag, Wiesbaden 2011, S. 109.
} 
[5] Kepplinger, H. M., Die psychologischen Gesetze des modernen Skandals, [w:] http://www.welt.de/debatte/kommentare/article13901048/Die-

psychologischen-Gesetze-des-modernen-Skandals.html 2012 (04.12.2016).

[6] Landwehr, I., Unfundiert, tendenziös und unnötig verletzend. GeenStijl - Das Medium ist der Skandal, [w:] Skandale. Strukturen und Strategien öffentlicher Aufmerksamkeitserzeugung, red. Bulkow, K., Petersen, C., VS Verlag, Wiesbaden 2011, S. 227-246.

[7] Weber, P., Determinanten von Skandalisierung in der politischen Auslandsberichterstattung: Eine empirische Analyse, [w:] Skandale. Strukturen und Strategien öffentlicher Aufmerksamkeitserzeugung, red. Bulkow, K., Petersen, C., VS Verlag, Wiesbaden 2011, S. 105-127.

[8] Volmert, J., ,Flick. Die gekaufte Republik“. Der Skandal, der die Republik erschütterte?, [w:] Skandale. Strukturen und Strategien öffentlicher Aufmerksamkeitserzeugung, red. Bulkow, K., Petersen, C., VS Verlag, Wiesbaden 2003, S. 22-46.

\section{MIĘDZY MEDIATYZACJĄ A SKANDALIZACJĄ. DETRONIZACJA IKONY POLSKIEGO REPORTAŻU}

Skandale były i są atrakcyjnym tematycznie materiałem dla mediów, ponieważ z jednej strony ujawniają łamanie norm i zasad społecznych, demaskują winnych, detronizując negatywnych bohaterów, $\mathrm{z}$ drugiej zaś stwarzają dziennikarzom okazję do medialnej autoprezentacji a nawet autoreklamy, gdyż pozwalają im wcielać się w rolę sprawiedliwych, stojących na straży zasad moralnych, dążących do normalizacji sytuacji wywołanej skandalem. $\mathrm{W}$ istniejących typologiach skandali w mediach mówi się ogólnie o skandalach mediatyzowanych, czyli komunikowanych medialnie oraz skandalach medialnych, w których głównymi skandalistami są same media. Wydaje się, że w przestrzeni medialnej funkcjonują także ich formy mieszane oscylujące pomiędzy oboma ich rodzajami. Na przykładzie skandalu wokół biografii Ryszarda Kapuścińskiego autorstwa jego redakcyjnego kolegi Artura Domosławskiego i sposobu ukazania reportera w niemieckiej prasie podjęta zostanie próba zanalizowania takiego właśnie skandalu mieszanego. Kolejnym pytaniem, z którym spróbują zmierzyć się autorki artykułu, będzie kwestia, czy prasa jedynie przekazuje, relacjonuje skandale swojej publiczności, czy może jednak ma tendencję do konstruowania 'własnych" skandali. Jednym z głównych celów skandalu wokół wspomnianej biografii była, jak można wnioskować z zebranego korpusu, medialna detronizacja pewnego społecznego autorytetu, jakim był i pozostaje mistrz reportażu - Ryszard Kapuściński. Interesujące w tym kontekście wydaje się przeanalizowanie, w jakim stopniu do tego demontażu przyczyniła się prasa niemiecka, 'relacjonując' skandal dyskutowany w Polsce. Czy pozostała neutralna, czy może jednak nieobiektywnie podeszła do skandalu wokół polskiego 'reportera stulecia'. Autorki artykułu zmierzą się z wymienionymi pytaniami, próbując znaleźć na nie odpowiedzi.

Slowa kluczowe: Ryszard Kapuściński, biografia Domosławskiego, skandal medialny, demontaż postaci, profilowanie w mediach.

\section{BETWEEN A MEDIATISED SCANDAL AND A MEDIA SCANDAL.} DISMANTLING A REPORTER ICON

Scandals have been and are topically attractive material for the media, because on one hand, they reveal breaches of social norms and rules, expose the guilty ones and dethrone negative protagonists. On the other hand, they create a possibility for the journalists for selfpresentation or even self-promotion, because they allow them to take the role of the righteous; ones who protect moral principles and those who want that the situation triggered by the scandal goes back to normal. In the existing typologies of media scandals, one 
generally talks about mediatised scandals - ones, which are communicated through the media, and media scandals - ones, in which the main scandalmonger is the media itself. It seems that mixed forms, oscillating between these two types, exist as well in the media sphere. An analysis of such a mixed form will be attempted on the basis of the scandal around the biography of Ryszard Kapuściński, written by his editorial colleague Artur Domosławski, and the portrayal of the reporter in the German press. Another aspect, which the authors of this article will deal with, is the question of whether the press only presents or reports on the scandals to its audience, or has a tendency of creating its 'own' scandals. As can be inferred from the collected corpus, one of the main aims of the scandal surrounding the mentioned biography was dethroning the kind of social authority, which was and still is the master of reportage, Ryszard Kapuściński. Within this context, it seems interesting to analyse how the German press contributed to the dismantling of an icon when it 'reported' on the scandal discussed in Poland. Did it remain neutral, or maybe it treated the scandal around the Polish 'reporter of the century' in a biased way? The authors of this article will deal with these questions and try to find answers to them.

Keywords: Ryszard Kapusciński, biography written by Domosławski, media scandal, dismantling of a character, profiling in the media.

DOI:10.7862/rz.2017.hss.5

Przesłano do redakcji: styczeń 2017

Przyjęto do druku: marzec 2017 
